\title{
ANALYSIS OF POSSIBILITY OF GROWTH OF SEVERAL EPITAXIAL LAYERS SIMULTANEOUSLY IN GAS PHAS- ES FRAMEWORK ONE TECHNOLOGICAL PROCESS. ON POSSIBILITY TO CHANGE PROPERTIES OF EPI-
} TAXIAL LAYERS

\author{
E.L. Pankratov and E.A. Bulaeva \\ Nizhny Novgorod State University, 23 Gagarin avenue, \\ Nizhny Novgorod, 603950, Russia
}

\begin{abstract}
We analyzed nonlinear model with varying in space and time coefficients of growth of epitaxial layers from gas phase in a vertical reactor with account native convection. We formulate several conditions to increase homogeneity of epitaxial layers with varying of technological process parameters.
\end{abstract}

\section{KEYWORDS}

Gas phase epitaxy; native convection; increasing of homogeneity of films; analytical approach for modeling

\section{INTRODUCTION}

In the present time different heterostructures are using to manufacture solid state electronic devices. The most common methods for growing heterostructures are epitaxy from gas and liquid phase, magnetron sputtering, molecular beam epitaxy. The are many experimental works describe manufacturing and growth of heterostructures [1-11]. At the same time essentially smaller quantity of works describes prognosis of epitaxy processes [12]. Main aim of the present paper is prognosis changing of properties of growing of epitaxial layers with changing of parameters of technological process with account native convection.

Framework the paper we consider gas phase epitaxy vertical reactor (see Fig. 1). The reactor consist of an external casing, keeper of substrate with a substrate and a spiral around the casing in area of the substrate to generate induction heating to activate chemical reactions of decay of reagents and to growth of the epitaxial layer. A mixture of gas reagents and gas-carrier inputs into inlet of the reactor from Fig. 1. Our main aim framework the paper is analysis of properties of growing of epitaxial layers on growth parameters with account native convection. 


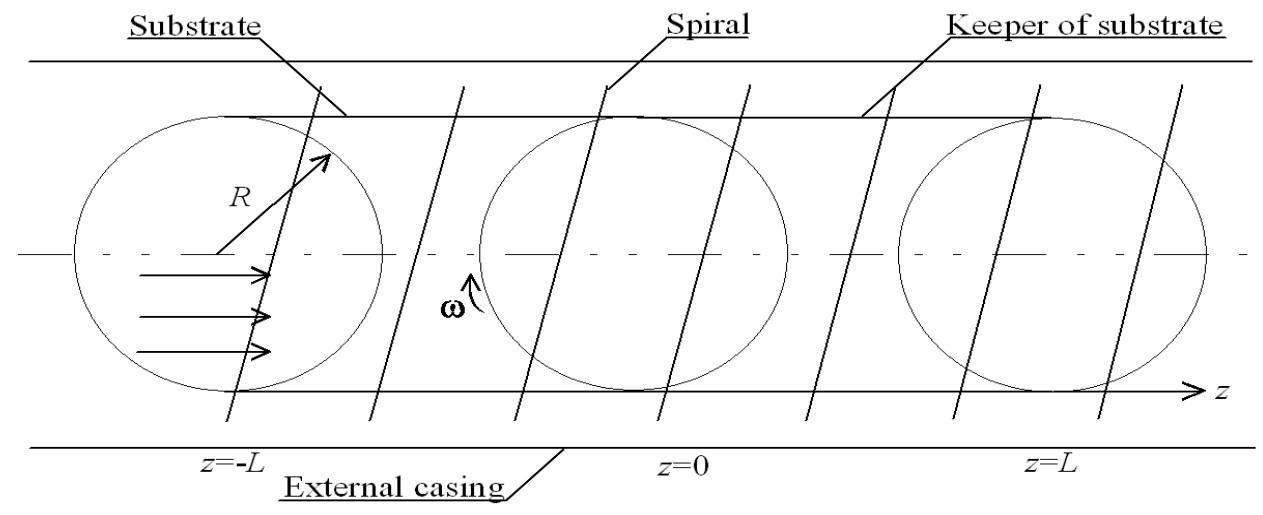

Fig. 1. Scheme of a reactor for gas phase epitaxy in area of reaction zone

\section{Method OF Solution}

We solve our aim by calculation and analysis of distribution of temperature in space and time. We calculate the distribution as solution of the following equation [13]

$$
\begin{aligned}
c \frac{\partial T(r, \varphi, z, t)}{\partial t}=p(r, \varphi, z, t)+\operatorname{div}\{ & \lambda \cdot \operatorname{grad}[T(r, \varphi, z, t)]- \\
& -[\vec{v}(r, \varphi, z, t)-\overrightarrow{\bar{v}}(r, \varphi, z, t)] \cdot c(T) \cdot T(r, \varphi, z, t) \cdot C(r, \varphi, z, t)\} .
\end{aligned}
$$

Here vector $\vec{v}$ describes speed of flow of mixture of gases; $c$ is the heat capacity; $T(r, \varphi, z, t)$ is the spatio-temporal distribution of temperature; $p(r, \varphi, z, t)$ is the density of power in the system substrate - keeper of substrate; $r, \varphi, z$ and $t$ are the cylindrical coordinates and time; $C(r, \varphi, z, t)$ is the spatio-temporal distribution of concentration of mixture of gases; $\lambda$ is the heat conductivity. Value of heat conductivity could be determine by the following relation: $\lambda=\bar{v} \bar{l} c_{v} \rho / 3$, where $\bar{v}$ is the modulus of mean squared speed of the gas molecules. The modulus is equal to following square root $\bar{v}=\sqrt{2 k T / m}$, parameter $\bar{l}$ describes the average free path of gas molecules between collisions, parameter $c_{v}$ describes the specific heat at constant volume, parameter $\rho$ describes the density of gas.

We solve the considered boundary problem with account moving of gases mixture. We calculate the spatio-temporal distribution of speed of mixture by coupling solving the equation of NavierStokes and the second Fourier law. We also assume that (i) radius of keeper of substrate $R$ very large in comparison with thickness of diffusion and near-boundary layers, (ii) stream of gas is laminar. In this situation required equations to describe spatio-temporal distributions of temperature and concentration of mixture of gases

$$
\begin{gathered}
\frac{\partial \vec{v}}{\partial t}+(\vec{v} \cdot \nabla) \vec{v}=-\nabla\left(\frac{P}{\rho}\right)+v \Delta \vec{v}, \\
\frac{\partial C(r, \varphi, z, t)}{\partial t}=\operatorname{div}\{D \cdot \operatorname{grad}[C(r, \varphi, z, t)]-[\vec{v}(r, \varphi, z, t)-\overrightarrow{\bar{v}}] \cdot C(r, \varphi, z, t)\} .
\end{gathered}
$$

Here parameter $D$ describes diffusion coefficient of mixture of gases (gases-carrier and gas-reagents); $P$ is the pressure; $\rho$ is the density of mixture of gases; $v$ is the kinematic viscosity. 
Framework the paper we consider regime of the limiting flow. In this situation all forthcoming to the disk molecules of deposit material are deposing on the substrate, flow is homogenous and one dimension. In this case boundary and initial conditions could be written as

$$
\begin{gathered}
C(r, \varphi,-L, t)=C_{0}, C(r, \varphi, 0, t)=0, C(r, 0, z, t)=C(r, 2 \pi, z, t), C(r, \varphi, z, 0)=C_{0} \delta(z+L), C(0, \varphi, z, t) \neq \infty, \\
\left.\frac{\partial C(r, \varphi, z, t)}{\partial r}\right|_{r=R}=0,\left.\frac{\partial C(r, \varphi, z, t)}{\partial \varphi}\right|_{\varphi=0}=\left.\frac{\partial C(r, \varphi, z, t)}{\partial \varphi}\right|_{\varphi=2 \pi},-\left.\lambda \frac{\partial T(r, \varphi, z, t)}{\partial r}\right|_{r=R}=\sigma T^{4}(R, \varphi, z, t), \\
\left.\frac{\partial T(r, \varphi, z, t)}{\partial \varphi}\right|_{\varphi=0}=\left.\frac{\partial T(r, \varphi, z, t)}{\partial \varphi}\right|_{\varphi=2 \pi},-\left.\lambda \frac{\partial T(r, \varphi, z, t)}{\partial z}\right|_{z=-L}=\sigma T^{4}(r, \varphi,-L, t),\left.\frac{\partial v_{r}(r, \varphi, z, t)}{\partial r}\right|_{r=0}=0, \\
\left.\frac{\partial v_{\varphi}(r, \varphi, z, t)}{\partial \varphi}\right|_{\varphi=0}=\left.\frac{\partial v_{\varphi}(r, \varphi, z, t)}{\partial \varphi}\right|_{\varphi=2 \pi},\left.\frac{\partial v_{\varphi}(r, \varphi, z, t)}{\partial \varphi}\right|_{\varphi=0}=\left.\frac{\partial v_{\varphi}(r, \varphi, z, t)}{\partial \varphi}\right|_{\varphi=2 \pi},\left.\frac{\partial v_{r}(r, \varphi, z, t)}{\partial r}\right|_{r=R}=0,(4) \\
-\left.\lambda \frac{\partial T(r, \varphi, z, t)}{\partial z}\right|_{z=L}=\sigma T^{4}(r, \varphi, z, t), T(r, 0, z, t)=T(r, 2 \pi, z, t), T(r, \varphi, z, 0)=T_{r}, T(0, \varphi, z, t) \neq \infty, v_{r}(r, \varphi, \\
-L, t)=0, v_{r}(r, \varphi, 0, t)=0, v_{r}(r, \varphi, L, t)=0, v_{r}(r, 0, z, t)=v_{r}(r, 2 \pi, z, t), v_{r}(0, \varphi, z, t) \neq \infty, v_{\varphi}(r, \varphi, 0, t)=\omega r, \\
v_{\varphi}(r, \varphi,-L, t)=0, v_{\varphi}(r, \varphi, L, t)=0, v_{\varphi}(r, 0, z, t)=v_{\varphi}(r, 2 \pi, z, t), v_{\varphi}(0, \varphi, z, t) \neq \infty, v_{z}(r, \varphi,-L, t)=V_{0}, v_{z}(r, \varphi, L, t)=0, \\
v_{z}(r, \varphi, 0, t)=\bar{v}_{z}, v_{z}(r, 0, z, t)=v_{z}(r, 2 \pi, z, t), v_{z}(0, \varphi, z, t) \neq \infty, v_{r}(r, \varphi, z, 0)=0, v_{\varphi}(r, \varphi, z, 0)=0, v_{z}(r, \varphi,-L, 0)=V_{0},
\end{gathered}
$$

where $\sigma=5,67 \cdot 10^{-8} \mathrm{~W} \cdot \mathrm{m}^{-2} \cdot \mathrm{K}^{-4}, T_{r}$ is the room temperature, $\omega$ is the frequency of rotation of substrate. We assume, that velocity of mixture of gases at $z=L$ has been chosen as minimal to decrease energy cost during growth of heterostructure.

Equations for components of velocity of flow with account cylindrical system of coordinate could be written as

$$
\begin{gathered}
\frac{\partial v_{r}}{\partial t}=-v_{r} \frac{\partial v_{r}}{\partial r}-\frac{v_{\varphi}}{r} \frac{\partial v_{\varphi}}{\partial \varphi}-v_{z} \frac{\partial v_{z}}{\partial z}+v\left(\frac{\partial^{2} v_{r}}{\partial r^{2}}+\frac{\partial^{2} v_{r}}{\partial r \partial z}-\frac{\partial^{2} v_{r}}{\partial z^{2}}+\frac{\partial^{2} v_{z}}{\partial r \partial}\right)-\frac{\partial}{\partial r}\left(\frac{P}{\rho}\right) \\
\frac{\partial v_{\varphi}}{\partial t}=-v_{r} \frac{\partial v_{r}}{\partial r}-\frac{v_{\varphi}}{r} \frac{\partial v_{\varphi}}{\partial \varphi}-v_{z} \frac{\partial v_{z}}{\partial z}+ \\
\quad+v\left(\frac{1}{r} \frac{\partial^{2} v_{r}}{\partial r \partial \varphi}+\frac{2}{r^{2}} \frac{\partial^{2} v_{\varphi}}{\partial \varphi^{2}}-\frac{1}{r^{2}} \frac{\partial^{2} v_{r}}{\partial \varphi \partial z}+\frac{\partial^{2} v_{\varphi}}{\partial z^{2}}\right)-\frac{1}{r} \frac{\partial}{\partial \varphi}\left(\frac{P}{\rho}\right) \\
\frac{\partial v_{z}}{\partial t}=-v_{r} \frac{\partial v_{r}}{\partial r}-\frac{v_{\varphi}}{r} \frac{\partial v_{\varphi}}{\partial \varphi}-v_{z} \frac{\partial v_{z}}{\partial z}+v\left(\frac{\partial^{2} v_{r}}{\partial z^{2}}+\frac{\partial^{2} v_{z}}{\partial r^{2}}+\frac{1}{r^{2}} \frac{\partial^{2} v_{z}}{\partial \varphi^{2}}\right)-\frac{\partial}{\partial z}\left(\frac{P}{\rho}\right) .
\end{gathered}
$$

We solve the above equations by using of method of averaging of function corrections [14-19]. The solution is presented in Appendix.

We calculate all required values (speed of stream of mixture of materials in gas phase: gases-reagents and gas-carrier; concentration of mixture of gases; temperature of growth) framework the second-order approximation framework method of averaging of function corrections. Recently we obtain, that the second-order approximation gives enough good approximation to make qualitative analysis of obtained solution and to obtain some quantitative results. These approximations are presented in Appendix.

To solve Eqs.(1) and (3) we re-write them by using cylindrical system of coordinate 


$$
\begin{gathered}
c \frac{\partial T(r, \varphi, z, t)}{\partial t}=\lambda\left[\frac{\partial^{2} T(r, \varphi, z, t)}{\partial r^{2}}+\frac{1}{r^{2}} \frac{\partial^{2} T(r, \varphi, z, t)}{\partial \varphi^{2}}+\frac{\partial^{2} T(r, \varphi, z, t)}{\partial z^{2}}\right]-c \cdot \frac{\partial}{\partial r}\{C(r, \varphi, z, t) . \\
\left.\cdot T(r, \varphi, z, t) \cdot\left[v_{r}(r, \varphi, z, t)-\bar{v}_{r}(r, \varphi, z, t)\right]\right\}-\frac{c}{r} \frac{\partial}{\partial \varphi}\left\{\left[v_{\varphi}(r, \varphi, z, t)-\bar{v}_{\varphi}(r, \varphi, z, t)\right] \cdot C(r, \varphi, z, t) .\right. \\
\cdot T(r, \varphi, z, t)\}-c \cdot \frac{\partial}{\partial z}\left\{\left[v_{z}(r, \varphi, z, t)-\bar{v}_{z}(r, \varphi, z, t)\right] \cdot C(r, \varphi, z, t) \cdot T(r, \varphi, z, t)\right\}+p(r, \varphi, z, t), \quad(12) \\
\frac{\partial C(r, \varphi, z, t)}{\partial t}=\frac{1}{r} \frac{\partial}{\partial r}\left[r D \frac{\partial C(r, \varphi, z, t)}{\partial r}\right]+\frac{1}{r^{2}} \frac{\partial}{\partial \varphi}\left[D \frac{\partial C(r, \varphi, z, t)}{\partial \varphi}\right]+\frac{\partial}{\partial z}\left[D \frac{\partial C(r, \varphi, z, t)}{\partial z}\right]- \\
-\frac{1}{r} \frac{\partial}{\partial r}\left\{r C(r, \varphi, z, t) \cdot\left[v_{r}(r, \varphi, z, t)-\bar{v}_{r}(r, \varphi, z, t)\right]\right\}-\frac{1}{r} \frac{\partial}{\partial \varphi}\left\{r C(r, \varphi, z, t) \cdot\left[v_{\varphi}(r, \varphi, z, t)-\bar{v}_{\varphi}(r, \varphi, z, t)\right]\right\}- \\
-\frac{\partial}{\partial z}\left\{C(r, \varphi, z, t) \cdot\left[v_{z}(r, \varphi, z, t)-\bar{v}_{z}(r, \varphi, z, t)\right]\right\} .
\end{gathered}
$$

\section{DisCUSSION}

In this paper we analyzed spatio-temporal distributions of gas and temperature during growth of epitaxial layers from gas phase to formulate conditions for improvement of properties of epitaxial layers. Fig. 2 shows concentration of mixture of reagents near epitaxial layer on frequency of rotation of substrate. Curve 1 describes dependence of concentration of reagents on the frequency of rotation of substrate at atmospheric pressure without taking into account native convection (at low temperature of growth one can neglect by native convection). Curve 2 describes dependence of concentration of reagents on the frequency of rotation of substrate at low pressure $(10 \%$ of atmospheric pressure) without taking into account native convection. Curve 3 describes dependence of concentration of reagents on the frequency of rotation of substrate at atmospheric pressure with account native convection. Increasing of temperature of growth leads to increasing of influence of native convection on growth process. The Fig.2 shows, that increasing of frequency of rotation of substrate gives a possibility to increase homogeneity of distribution of concentration deposited on the substrate material on radius of the substrate. The Fig. 3 shows, that concentration of concentration of material of epitaxial layer on diffusion coefficient $D$. Curve 1 describes dependence of concentration of reagents on the diffusion coefficient at atmospheric pressure without taking into account native convection. Curve 2 describes dependence of concentration of reagents on the diffusion coefficient at low pressure (10\% of atmospheric pressure) without taking into account native convection. Curve 3 describes dependence of concentration of reagents on the diffusion coefficient at atmospheric pressure with account native convection. One can find from the figure, that monotonous decreasing of the considered concentration. With increasing of diffusion coefficient one can find increasing of speed of transport of gases and decreasing of quantity of reagents in reaction zone due to higher speed of gases. In this situation one can find decreasing of speed of growth of epitaxial layer. Value of diffusion coefficient will be smaller with decreasing of temperature of grown. At the same time decreasing of growth temperature leads to deceleration of chemical reaction. 
International Journal on Organic Electronics (IJOE) Vol.6, No.1, January 2017

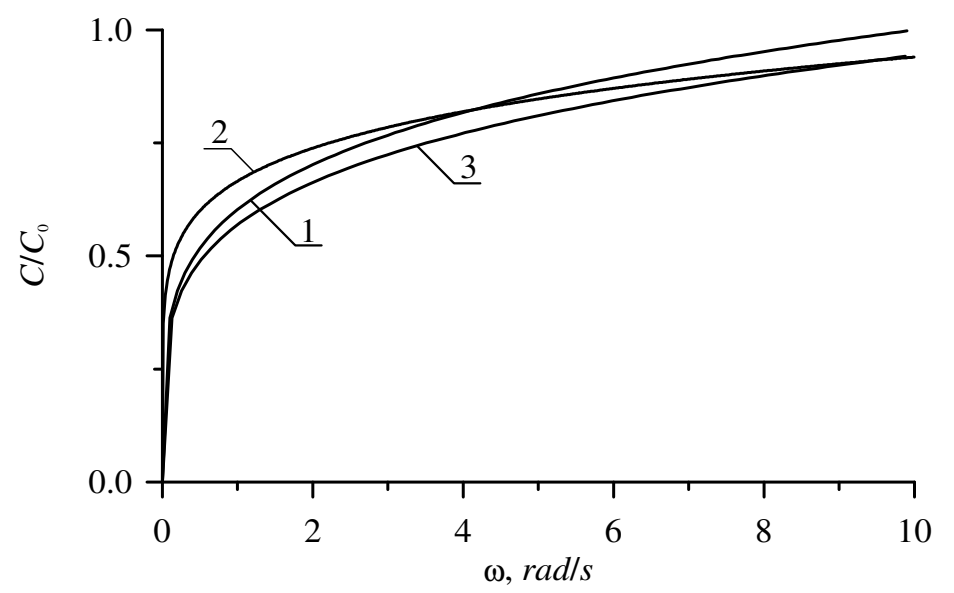

Fig. 2. Dependences of concentration of gaseous mixture on frequency of rotation of substrate

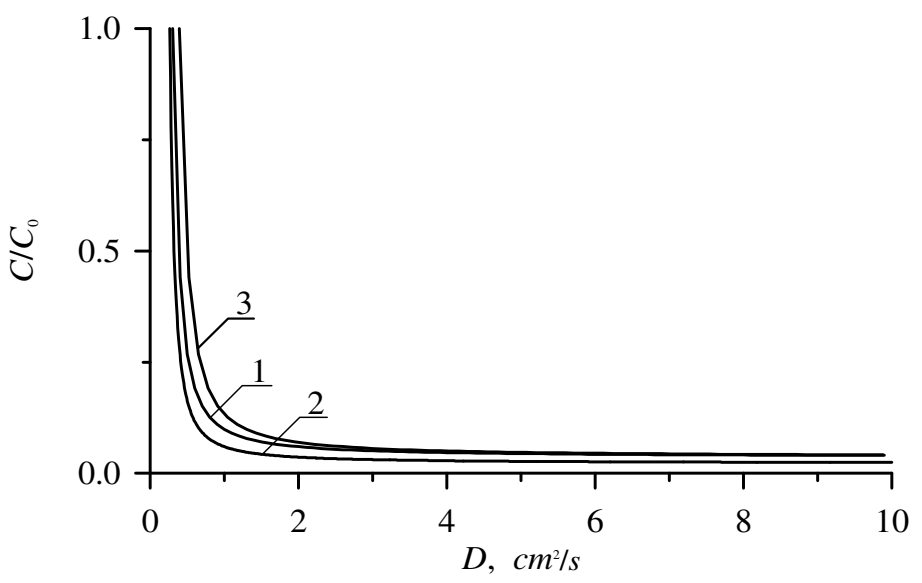

Fig. 3. Dependences of concentration of gaseous mixture on diffusion coefficient

Dependence of gaseous mixture concentration on kinematical viscosity $v$ is presented on Fig. 4. Designations curves in this figure are the same as in the previous two. Increasing of the viscosity leads to decreasing of the concentration due to deceleration of gas transport. Fig. 5 shows dependences of the considered gaseous mixture concentration on inlet speed of gases $V_{0}$ is presented on Fig. 5. Curve 1 describes dependence of concentration of reagents on the speed $V_{0}$ at atmospheric pressure without taking into account native convection. Curve 2 describes dependence of concentration of reagents on the speed $V_{0}$ at low pressure (10\% of atmospheric pressure) without taking into account native convection. Curve 3 describes dependence of concentration of reagents on the speed $V_{0}$ at atmospheric pressure with account native convection. Increasing of the parameter $V_{0}$ gives a possibility to increasing the concentration of reagents in reaction zone and to increase speed of growth of epitaxial layers. 


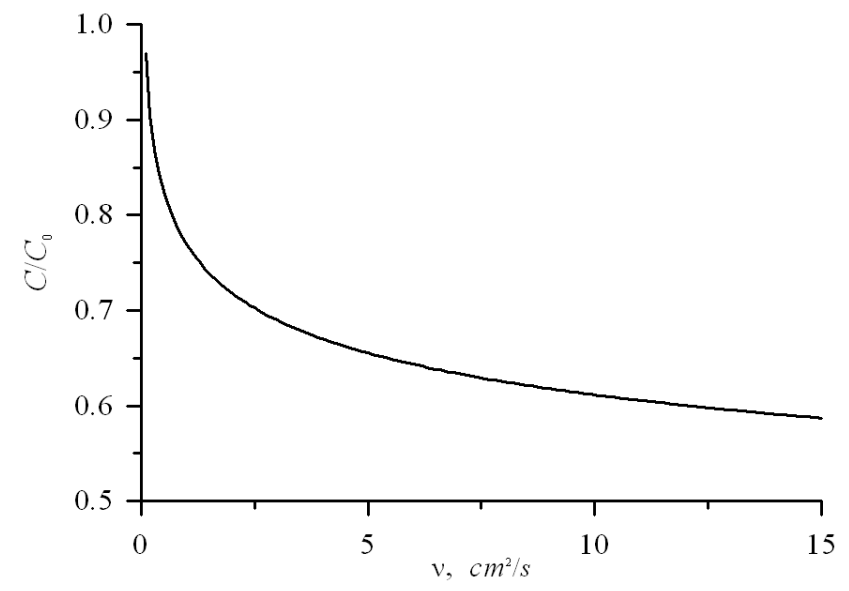

Fig. 4. Dependence of concentration of mixture of gases on kinematical viscosity $v$

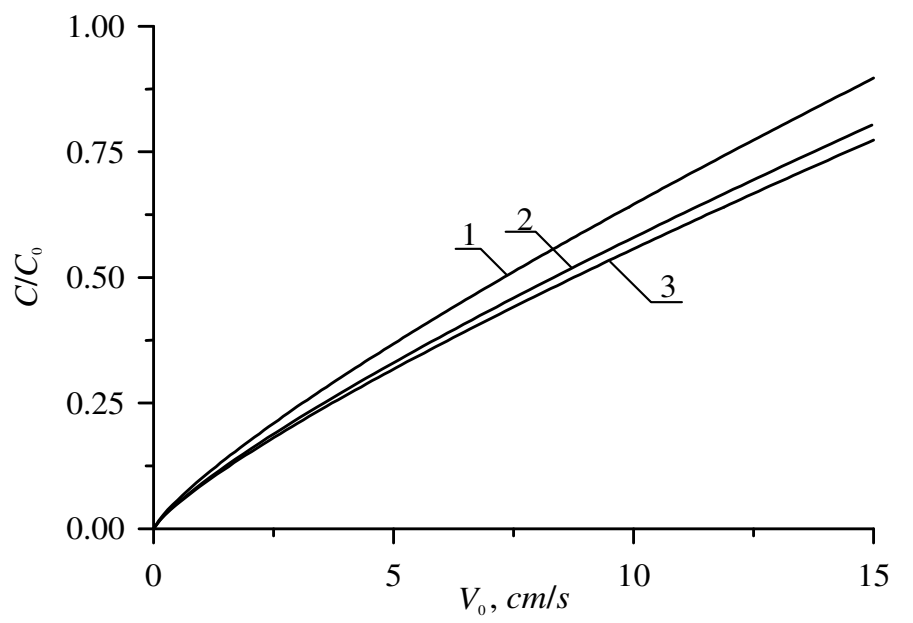

Fig. 5. Dependences of concentration of mixture of gases on inlet speed

Analysis of spatio-temporal distribution of gaseous mixture concentration shows, that decreasing of pressure in the reactor leads to reducing the inertness of the considered processes of mass and heat transport. Native convection leads to deceleration of growth of epitaxial layers. It should be noted, that it is necessary to choose value of power of induction heating of growth area to compensate heat loss due to convective heat [21]. In this case heating time $\vartheta$ could be estimated framework recently introduced approach [22] and could be calculated as $\vartheta \approx(6 \pi-1) R^{2} / 24 \lambda_{0}$, where $\lambda_{0}$ is the average value of heat conductivity. In this case power, which is necessary to compensate losing of heat during manufacturing of epitaxial layer, could be estimated by using the following relation: $\int_{0}^{R} r \cdot p(r, \varphi, z, t) d r \approx \sigma \cdot T^{4}(R, \varphi, z, t)+\Theta \cdot v_{z}(R, \varphi, z, t) / 4 \pi L R^{2}$.

\section{Conclusions}

In the present paper we analyzed dependences of dynamics of mass and heat transport in reactors for epitaxy from gas phase on physical and technological parameters. We compare growth regimes at atmospheric and at low pressure and analyzed influence of native convection on growth of epitaxial layers. Accounting the calculated results it have been formulated recommendations to improve properties of epitaxial layers. 
International Journal on Organic Electronics (IJOE) Vol.6, No.1, January 2017

\section{ACKNOWLEDGEMENTS}

This work is supported by the agreement of August 27, 2013 № 02.B.49.21.0003 between The Ministry of education and science of the Russian Federation and Lobachevsky State University of Nizhni Novgorod.

\section{REFERENCES}

1. I.P. Stepanenko. Basis of microelectronics. Moscow, Soviet radio (in Russian), 1980.

2. V.G. Gusev, Yu.M. Gusev. Electronics. Moscow, Higher School (in Russian), 1991.

3. V.I. Lachin, N.S. Savelov. Electronics. Rostov-on-Don, Phenics, 2001.

4. A.A. Vorob'ev, V.V. Korabl'ev, S.Yu. Karpov. The use of magnesium to dope gallium nitride obtained by molecular-beam epitaxy from activated nitrogen. Semiconductors. Vol. 37 (7). P. 98 (2003).

5. L.M. Sorokin, N.V. Veselov, M.P. Shcheglov, A.E. Kalmykov, A.A. Sitnikova, N.A. Feoktistov, A.V. Osipov, S.A. Kukushkin. Electron-microscopic investigation of a SiC/Si(111) structure obtained by solid phase epitaxy. Technical Physics Letters. Vol. 34 (22). P. 68 (2008).

6. V.V. Lundin, A.V. Sakharov, E.E. Zavarin, M.A. Sinitsin, A.E. Nikolaevb, G.A. Mikhailovsky, P.N. Brunkov, V.V. Goncharov, B.Ya. Ber, D.Yu. Kazantsev, A.F. Tsatsul'nikov. Effect of carrier gas and doping profile on the surface morphology of MOVPE grown heavily doped GaN:Mg layers. Semiconductors. Vol. 43 (7). P. 996 (2009).

7. Y.E. Bravo-García, P. Rodríguez-Fragoso, J.G. Mendoza-Alvarez, G. Gonzalezdela Cruz. Growth and characterization of InAsSb layers on GaSb substrates by liquid phase epitaxy. Mat. Sci. Sem. Proc. Vol. 40. P. 253-256 (2015).

8. Y. Li, L.E. Antonuk, Y. El-Mohri, Q. Zhao, H. Du, A. Sawant, Yi Wang. J. Appl. Phys. Vol. 99 (6). P. 064501 (2006).

9. A. Chakraborty, H. Xing, M.D. Craven, S. Keller, T. Mates, J. S. Speck, S.P. Den Baars, U.K. Mishra. Nonpolar $\alpha$-plane p-type GaN and p-n-junction diodes. J. Appl. Phys. Vol. 96 (8). P. 4494 (2004).

10. H. Taguchia, S. Miyakea, A. Suzukib, S. Kamiyamab, Y. Fujiwarac. Evaluation of crystallinity of GaN epitaxial layer after wafer dicing. Mat. Sci. Sem. Proc. Vol. 41. P. 89-61 (2016).

11. M. Mitsuhara, M. Ogasawara, H. Sugiura. Beryllium doping of InP during metalorganic molecular beam epitaxy using bismethylcyclopentadienyl-beryllium. J. Cryst. Growth. Vol. 183. P. 38 (1998).

12. R.A. Talalaev, E.V. Yakovleva, S.Yu. Karpova, Yu.N. Makarov. On low temperature kinetic effects in metal-organic vapor phase epitaxy of III-V compounds. J. Cryst. Growth. Vol. 230. P. $232(2001)$.

13. H.S. Carslaw, J.C. Jaeger. Conduction of heat in solids. - Oxford: At the Clarendon Press. 1964. $488 \mathrm{p}$.

14. Yu.D. Sokolov. About the definition of dynamic forces in the mine lifting. Applied Mechanics. Vol.1 (1). P. 23-35 (1955).

15. E.L. Pankratov. Decreasing of depth of p-n-junction in a semiconductor heterostructure by serial radiation processing and microwave annealing. J. Comp. Theor. Nanoscience. Vol.9 (1). P. 4149 (2012).

16. E.L. Pankratov, E.A. Bulaeva. Application of native inhomogeneities to increase compactness of vertical field-effect transistors. J. Comp. Theor. Nanoscience. Vol.10 (4). P. 888-893 (2013).

17. E.L. Pankratov, E.A. Bulaeva. Decreasing of quantity of radiation defects in an implantedjunction rectifiers by using overlayers. International Journal of Micro-Nano Scale Transport. Vol. 3 (3). P. 119-130 (2012).

18. E.L. Pankratov, E.A. Bulaeva. About controlling of regimes of heating during growth a heterostructures from gas phase. Universal Journal of Materials Science. Vol. 1 (4). P. 180-200 (2013).

19. E.L. Pankratov, E.A. Bulaeva. Reviews in Theoretical Science. Vol. 1 (1). P. 58-82 (2013).

20. G. Korn, T. Korn. Mathematical Handbook for scientists and engineers. Definitions, theorems and formulas for reference and review. Second edition. McGraw-Hill Book Company. New York, 1968. 
International Journal on Organic Electronics (IJOE) Vol.6, No.1, January 2017

21. E.L. Pankratov, E.A. Bulaeva. On prognosis of epitaxy from gas phase process for improvement of properties of films. 3D research. Vol. 6 (4). P. 46-56 (2015).

22. E.L. Pankratov, E.A. Bulaeva. Optimal Criteria to Estimate Temporal Characteristics of Diffusion Process in a Media with Inhomogenous and Nonstationary Parameters. Analysis of Influence of Variation of Diffusion Coefficient on Values of Time Characteristics. Reviews in Theoretical Science. Vol. 1 (3). P. 305-316 (2013).

\section{Authors:}

Pankratov Evgeny Leonidovich was born at 1977. From 1985 to 1995 he was educated in a secondary school in Nizhny Novgorod. From 1995 to 2004 he was educated in Nizhny Novgorod State University: from 1995 to 1999 it was bachelor course in Radiophysics, from 1999 to 2001 it was master course in Radiophysics with specialization in Statistical Radiophysics, from 2001 to 2004 it was PhD course in Radiophysics. From 2004 to 2008 E.L. Pankratov was a leading technologist in Institute for Physics of Microstructures. From 2008 to 2012 E.L. Pankratov was a senior lecture/Associate Professor of Nizhny Novgorod State University of Architecture and Civil Engineering. Now E.L. Pankratov is in his Full Doctor course in Radiophysical Department of Nizhny Novgorod State University. He has 140 published papers in area of his researches.

Bulaeva Elena Alexeevna was born at 1991. From 1997 to 2007 she was educated in secondary school of village Kochunovo of Nizhny Novgorod region. From 2007 to 2009 she was educated in boarding school "Center for gifted children". From 2009 she is a student of Nizhny Novgorod State University of Architecture and Civil Engineering (spatiality "Assessment and management of real estate"). At the same time she is a student of courses "Translator in the field of professional communication" and "Design (interior art)" in the University. E.A. Bulaeva was a contributor of grant of President of Russia (grant № MK548.2010.2). She has 75 published papers in area of her researches.

\section{Appendix}

We determine solution of system of equations for components of speed of mixture by using of method of averaging of function corrections. Framework this approach to determine the firstorder approximation of components of speed of flow of mixture of gases we replace of the required functions on their average values $v_{r} \rightarrow \alpha_{1 r}, v_{\varphi} \rightarrow \alpha_{1 \varphi}, v_{z} \rightarrow \alpha_{1 z}$ in right sides of equations of system (5). After the replacement we obtain equations for the first-order approximations of the components

$$
\frac{\partial v_{1 r}}{\partial t}=-\frac{\partial}{\partial r}\left(\frac{P}{\rho}\right), \frac{\partial v_{1 \varphi}}{\partial t}=-\frac{1}{r} \frac{\partial}{\partial \varphi}\left(\frac{P}{\rho}\right), \frac{\partial v_{1 z}}{\partial t}=-\frac{\partial}{\partial z}\left(\frac{P}{\rho}\right) .
$$

Solutions of the Eqs.(6) could be written as

$$
v_{1 r}=-\frac{\partial}{\partial r} \int_{0}^{t} \frac{P}{\rho} d \tau, v_{1 \varphi}=-\frac{1}{r} \frac{\partial}{\partial \varphi} \int_{0}^{t} \frac{P}{\rho} d \tau, v_{1 z}=-\frac{\partial}{\partial z} \int_{0}^{t} \frac{P}{\rho} d \tau .
$$

One can obtain the second-order approximations of components of speed of flow by replacement of the required functions on the following sums $v_{r} \rightarrow \alpha_{1 r}, v_{\varphi} \rightarrow \alpha_{1 \varphi}, v_{z} \rightarrow \alpha_{1 z}$. Approximations for the components could be written as

$$
\begin{aligned}
\frac{\partial v_{2 r}}{\partial t}=v\left(\frac{\partial^{2} v_{1 r}}{\partial r^{2}}+\frac{\partial^{2} v_{1 r}}{\partial r \partial z}-\frac{\partial^{2} v_{1 r}}{\partial z^{2}}+\frac{\partial^{2} v_{1 z}}{\partial r \partial z}\right) & -\frac{\partial}{\partial r}\left(\frac{P}{\rho}\right)- \\
& -\left(\alpha_{2 r}+v_{1 r}\right) \frac{\partial v_{1 r}}{\partial r}-\frac{\left(\alpha_{2 \varphi}+v_{1 \varphi}\right)}{r} \frac{\partial v_{1 r}}{\partial \varphi}-\left(\alpha_{2 z}+v_{1 z}\right) \frac{\partial v_{1 r}}{\partial z},(8 a)
\end{aligned}
$$




$$
\begin{aligned}
\frac{\partial v_{2 \varphi}}{\partial t}=v\left(\frac{1}{r} \frac{\partial^{2} v_{1 r}}{\partial r \partial \varphi}+\frac{2}{r^{2}} \frac{\partial^{2} v_{1 \varphi}}{\partial \varphi^{2}}-\frac{1}{r^{2}}\right. & \left.\frac{\partial^{2} v_{1 r}}{\partial \varphi \partial}+\frac{\partial^{2} v_{1 \varphi}}{\partial z^{2}}\right)-\frac{1}{r} \frac{\partial}{\partial \varphi}\left(\frac{P}{\rho}\right)- \\
& -\left(\alpha_{2 r}+v_{1 r}\right) \frac{\partial v_{1 \varphi}}{\partial r}-\frac{\left(\alpha_{2 \varphi}+v_{1 \varphi}\right)}{r} \frac{\partial v_{1 \varphi}}{\partial \varphi}-\left(\alpha_{2 z}+v_{1 z}\right) \frac{\partial v_{1 \varphi}}{\partial z}, \\
\frac{\partial v_{2 z}}{\partial t}=v\left(\frac{\partial^{2} v_{1 r}}{\partial z^{2}}+\frac{\partial^{2} v_{1 z}}{\partial r^{2}}+\frac{1}{r^{2}} \frac{\partial^{2} v_{1 z}}{\partial \varphi^{2}}\right) & -\frac{\partial}{\partial z}\left(\frac{P}{\rho}\right)- \\
& -\left(\alpha_{2 r}+v_{1 r}\right) \frac{\partial v_{1 z}}{\partial r}-\frac{\left(\alpha_{2 \varphi}+v_{1 \varphi}\right)}{r} \frac{\partial v_{1 z}}{\partial \varphi}-\left(\alpha_{2 z}+v_{1 z}\right) \frac{\partial v_{1 z}}{\partial z} .
\end{aligned}
$$

Integration of the above equations leads to the following result

$$
\begin{aligned}
v_{2 r}=v \int_{0}^{t}\left(\frac{\partial^{2} v_{1 r}}{\partial r^{2}}+\frac{\partial^{2} v_{1 r}}{\partial r \partial z}-\frac{\partial^{2} v_{1 r}}{\partial z^{2}}+\frac{\partial^{2} v_{1 z}}{\partial r \partial z}\right) d \tau-\frac{\partial}{\partial r}\left(\int_{0}^{t} \frac{P}{\rho} d \tau\right)- \\
-\int_{0}^{t}\left(\alpha_{2 r}+v_{1 r}\right) \frac{\partial v_{1 r}}{\partial r} d \tau-\int_{0}^{t} \frac{\left(\alpha_{2 \varphi}+v_{1 \varphi}\right)}{r} \frac{\partial v_{1 r}}{\partial \varphi} d \tau-\int_{0}^{t}\left(\alpha_{2 z}+v_{1 z}\right) \frac{\partial v_{1 r}}{\partial z} d \tau, \\
v_{2 \varphi}=v \int_{0}^{t}\left(\frac{1}{r} \frac{\partial^{2} v_{1 r}}{\partial r \partial \varphi}+\frac{2}{r^{2}} \frac{\partial^{2} v_{1 \varphi}}{\partial \varphi^{2}}-\frac{1}{r^{2}} \frac{\partial^{2} v_{1 r}}{\partial \varphi \partial z}+\frac{\partial^{2} v_{1 \varphi}}{\partial z^{2}}\right) d \tau-\frac{1}{r} \frac{\partial}{\partial \varphi}\left(\int_{0}^{t} \frac{P}{\rho} d \tau\right)- \\
-\int_{0}^{t}\left(\alpha_{2 r}+v_{1 r}\right) \frac{\partial v_{1 \varphi}}{\partial r} d \tau-\int_{0}^{t} \frac{\left(\alpha_{2 \varphi}+v_{1 \varphi}\right)}{r} \frac{\partial v_{1 \varphi}}{\partial \varphi} d \tau-\int_{0}^{t}\left(\alpha_{2 z}+v_{1 z}\right) \frac{\partial v_{1 \varphi}}{\partial z} d \tau, \\
v_{2 z}=v \int_{0}^{t}\left(\frac{\partial^{2} v_{1 r}}{\partial z^{2}}+\frac{\partial^{2} v_{1 z}}{\partial r^{2}}+\frac{1}{r^{2}} \frac{\partial^{2} v_{1 z}}{\partial \varphi^{2}}\right) d \tau-\frac{\partial}{\partial z}\left(\int_{0}^{t} \frac{P}{\rho} d \tau\right)- \\
-\int_{0}^{t}\left(\alpha_{2 r}+v_{1 r}\right) \frac{\partial v_{1 z}}{\partial r} d \tau-\int_{0}^{t} \frac{\left(\alpha_{2 \varphi}+v_{1 \varphi}\right)}{r}\left(\alpha_{2 \varphi}+v_{1 \varphi}\right) \frac{\partial v_{1 z}}{\partial \varphi} d \tau-\int_{0}^{t}\left(\alpha_{2 z}+v_{1 z}\right) \frac{\partial v_{1 z}}{\partial z} d \tau .
\end{aligned}
$$

We determine average values $\alpha_{2 r}, \alpha_{2 \varphi}, \alpha_{2 z}$ by the following relations

$$
\begin{gathered}
\alpha_{2 r}=\frac{1}{\pi \Theta R^{2} L} \int_{0}^{\Theta} \int_{0}^{R} r \int_{0}^{2 \pi L} \int_{-L}^{L}\left(v_{2 r}-v_{1 r}\right) d z d \varphi d r d t, \alpha_{2 \varphi}=\frac{1}{\pi \Theta R^{2} L} \iint_{0}^{\Theta} \int_{0}^{R} r \int_{0}^{2 \pi L} \int_{-L}^{L}\left(v_{2 \varphi}-v_{1 \varphi}\right) d z d \varphi d r d t \\
\alpha_{2 z}=\frac{1}{\pi \Theta R^{2} L} \int_{0}^{\Omega} \int_{0}^{R} r \int_{0}^{2 \pi L} \int_{-L}^{L}\left(v_{2 z}-v_{1 z}\right) d z d \varphi d r d t
\end{gathered}
$$

where $\Theta$ is the continuance of moving of mixture of gases through reactor. Substitution of both (the first- and the second-order) approximations of the required components of the considered speed into the relation (9) give us possibility to obtain system of equations to determine required average values

$$
\left\{\begin{array}{l}
A_{1} \alpha_{2 r}+B_{1} \alpha_{2 \varphi}+C_{1} \alpha_{2 z}=D_{1} \\
A_{2} \alpha_{2 r}+B_{2} \alpha_{2 \varphi}+C_{2} \alpha_{2 z}=D_{2} \\
A_{3} \alpha_{2 r}+B_{3} \alpha_{2 \varphi}+C_{3} \alpha_{2 z}=D_{3}
\end{array}\right.
$$


Here $\quad A_{1}=1+\int_{0}^{\Theta}(\Theta-t) \int_{0}^{R} r \int_{0}^{2 \pi L} \int_{-L}^{L} \frac{\partial v_{1 r}}{\partial r} d z d \varphi d r d t, \quad B_{1}=\int_{0}^{\Theta}(\Theta-t) \int_{0}^{R} \int_{0}^{2 \pi} \int_{-L}^{L} \frac{\partial v_{1 r}}{\partial \varphi} d z d \varphi d r d t, \quad C_{1}=C_{2}=$ $=\frac{\pi}{2} \Theta^{2} R^{2} V_{0}, D_{1}=v \int_{0}^{\Theta}(\Theta-t) \int_{0}^{R} r \int_{0}^{2 \pi} \int_{-L}^{L}\left(\frac{\partial^{2} v_{1 r}}{\partial r^{2}}+\frac{\partial^{2} v_{1 r}}{\partial r \partial z}-\frac{\partial^{2} v_{1 r}}{\partial z^{2}}+\frac{\partial^{2} v_{1 z}}{\partial r \partial z}\right) d z d \varphi d r d t-\frac{\pi}{8} \Theta^{2} R^{2} \times$ $\times V_{0}^{2}-\int_{0}^{\Theta}(\Theta-t) \int_{0}^{R} r \int_{0}^{2 \pi} \int_{-L}^{L} v_{1 r} \frac{\partial v_{1 r}}{\partial r} d z d \varphi d r d t-\int_{0}^{\Theta}(\Theta-t) \int_{0}^{R} \int_{0}^{2 \pi} \int_{-L}^{L} v_{1 \varphi} \frac{\partial v_{1 r}}{\partial \varphi} d z d \varphi d r d t, A_{2}=\int_{0}^{\Theta}(\Theta-t) \times$ $\times \int_{0}^{R} r \int_{0}^{2 \pi} \int_{-L}^{L} \frac{\partial v_{1 r}}{\partial r} d z d \varphi d r d t, \quad B_{2}=1+\int_{0}^{\Theta}(\Theta-t) \int_{0}^{R} \int_{0}^{2 \pi} \int_{-L}^{L} \frac{\partial v_{1 r}}{\partial \varphi} d z d \varphi d r d t, \quad D_{2}=v \int_{0}^{\Theta}(\Theta-t) \int_{0}^{R} r \int_{0}^{2 \pi} \int_{-L}^{L}\left(\frac{1}{r} \times\right.$ $\left.\times \frac{\partial^{2} v_{1 r}}{\partial r \partial \varphi}+\frac{2}{r^{2}} \frac{\partial^{2} v_{1 \varphi}}{\partial \varphi^{2}}-\frac{1}{r^{2}} \frac{\partial^{2} v_{1 r}}{\partial \varphi \partial z}+\frac{\partial^{2} v_{1 \varphi}}{\partial z^{2}}\right) d z d \varphi d r d t-\frac{\pi}{8} \Theta^{2} R^{2} V_{0}^{2}-\int_{0}^{\Theta}(\Theta-t) \int_{0}^{R} r \int_{0}^{2 \pi} \int_{-L}^{L} \frac{\partial v_{1 r}}{\partial r} \times$ $\times v_{1 r} d z d \varphi d r d t-\int_{0}^{\Theta}(\Theta-t) \int_{0}^{R} \int_{0}^{2 \pi} \int_{-L}^{L} v_{1 \varphi} \frac{\partial v_{1 r}}{\partial \varphi} d z d \varphi d r d t, A_{3}=\int_{0}^{\Theta}(\Theta-t) \int_{0}^{R} r \int_{0}^{2 \pi} \int_{-L}^{L} \frac{\partial v_{1 z}}{\partial r} d z d \varphi d r d t$, $B_{3}=\int_{0}^{\Theta}(\Theta-t) \int_{0}^{R} r \int_{0}^{2 \pi} \int_{-L}^{L} \frac{\partial v_{1 z}}{\partial \varphi} d z d \varphi d r d t, C_{3}=1+\frac{\pi}{2} \Theta^{2} R^{2} V_{0}, D_{3}=v \int_{0}^{\Theta}(\Theta-t) \int_{0}^{R} r \int_{0}^{2 \pi} \int_{-L}^{L}\left(\frac{\partial^{2} v_{1 r}}{\partial z^{2}}+\frac{\partial^{2} v_{1 z}}{\partial r^{2}}+\right.$ $\left.+\frac{1}{r^{2}} \frac{\partial^{2} v_{1 z}}{\partial \varphi^{2}}\right) d z d \varphi d r d t-\int_{0}^{\Theta}(\Theta-t) \int_{0}^{R} r \int_{0}^{2 \pi} \int_{-L}^{L} v_{1 r} \frac{\partial v_{1 z}}{\partial r} d z d \varphi d r d t-\int_{0}^{\Theta} \int_{0}^{R} r \int_{0}^{2 \pi L} \int_{-L}^{L} v_{1 \varphi} \frac{\partial v_{1 z}}{\partial \varphi} d z d \varphi d r \times$ $\times(\Theta-t) d t-\pi \Theta^{2} R^{2} V_{0}^{2} / 8$.

Solution of the above system of equations could be determined by standard approaches [19] and could be written as

$$
\alpha_{2 r}=\Delta_{r} / \Delta, \alpha_{2 \varphi}=\Delta_{\varphi} / \Delta, \alpha_{2 z}=\Delta_{z} / \Delta
$$

where $\Delta=A_{1}\left(B_{2} C_{3}-B_{3} C_{2}\right)-B_{1}\left(A_{2} C_{3}-A_{3} C_{2}\right)+C_{1}\left(A_{2} B_{3}-A_{3} B_{2}\right), \Delta_{r}=D_{1}\left(B_{2} C_{3}-B_{3} C_{2}\right)-\left(D_{2} C_{3}-\right.$ $\left.-D_{3} C_{2}\right) B_{1}+C_{1}\left(D_{2} B_{3}-D_{3} B_{2}\right), \Delta_{\varphi}=D_{1}\left(B_{2} C_{3}-B_{3} C_{2}\right)-B_{1}\left(D_{2} C_{3}-D_{3} C_{2}\right)+C_{1}\left(D_{2} B_{3}-D_{3} B_{2}\right)$, $\Delta_{z}=A_{1}\left(B_{2} D_{3}-B_{3} D_{2}\right)-B_{1}\left(A_{2} D_{3}-A_{3} D_{2}\right)+D_{1}\left(A_{2} B_{3}-A_{3} B_{2}\right)$.

We solve Eqs.(12) and (13) we used method of average of function corrections. We calculate the first- order approximations of the by replacement of them on their not yet known average values $\alpha_{1 T}$ and $\alpha_{1 C}$ in right sides of the above equations. Farther we used recently consider algorithm to obtain the first-order approximations of temperature and concentration of gas mixture

$$
\begin{gathered}
T_{1}(r, \varphi, z, t)=T_{r}+\int_{0}^{t} \frac{p(r, \varphi, z, \tau)}{c} d \tau-\alpha_{1 T} \alpha_{1 C} \int_{0}^{t} \frac{\partial\left[v_{r}(r, \varphi, z, \tau)-\bar{v}_{r}(r, \varphi, z, \tau)\right]}{\partial r} d \tau- \\
-\frac{\alpha_{1 T} \alpha_{1 C}}{r} \int_{0}^{t} \frac{\partial\left[v_{\varphi}(r, \varphi, z, \tau)-\bar{v}_{\varphi}(r, \varphi, z, \tau)\right]}{\partial \varphi} d \tau-\alpha_{1 T} \alpha_{1 C} \int_{0}^{t} \frac{\partial\left[v_{z}(r, \varphi, z, \tau)-\bar{v}_{z}(r, \varphi, z, \tau)\right]}{\partial z} d \tau, \\
C_{1}(r, \varphi, z, t)=C_{0}-\frac{\alpha_{1 C}}{r} \int_{0}^{t} \frac{\partial\left\{r\left[v_{r}(r, \varphi, z, \tau)-\bar{v}_{r}(r, \varphi, z, \tau)\right]\right\}}{\partial r} d \tau- \\
-\frac{\alpha_{1 C}}{r} \int_{0}^{t} \frac{\partial\left[v_{\varphi}(r, \varphi, z, \tau)-\bar{v}_{\varphi}(r, \varphi, z, \tau)\right]}{\partial \varphi} d \tau-\alpha_{1 C} \int_{0}^{t} \frac{\partial\left[v_{z}(r, \varphi, z, \tau)-\bar{v}_{z}(r, \varphi, z, \tau)\right]}{\partial z} d \tau .
\end{gathered}
$$

The above not yet known average values could be determined by the standard relations 
International Journal on Organic Electronics (IJOE) Vol.6, No.1, January 2017

$$
\begin{aligned}
& \alpha_{1 T}=\frac{1}{\pi \Theta R^{2} L} \int_{0}^{\Theta} \int_{0}^{R} r \int_{0}^{2 \pi} \int_{-L}^{L} T_{1}(r, \varphi, z, \tau) d z d \varphi d r d t, \\
& \alpha_{1 C}=\frac{1}{\pi \Theta R^{2} L} \int_{0}^{\Theta} \int_{0}^{R} r \int_{0}^{2 \pi} \int_{-L}^{L} C_{1}(r, \varphi, z, \tau) d z d \varphi d r d t .
\end{aligned}
$$

Substitution of the first-order approximations of temperature and concentration of gas mixture into relations (16) gives us the following results [20]

$$
\begin{gathered}
\alpha_{1 C}=C_{0} / L \cdot\left\{1+\frac{1}{\pi \Theta R L} \int_{0}^{\Theta}(\Theta-t) \int_{0}^{2 \pi} \int_{-L}^{L}\left[v_{r}(R, \varphi, z, t)-\bar{v}_{r}(R, \varphi, z, t)\right] d z d \varphi d t+\frac{\Theta V_{0}}{R L}\right\}, \\
\alpha_{1 T}=\left[T_{r}+\frac{1}{\pi \Theta R^{2} L} \int_{0}^{\Theta}(\Theta-t) \int_{0}^{R} r \int_{0}^{2 \pi} \int_{-L}^{L} \frac{p(r, \varphi, z, t)}{c} d z d \varphi d r d t\right]\left(1+\frac{C_{0}}{\pi \Theta R L^{2}}\left\{\int _ { 0 } ^ { \Theta } \int _ { 0 } ^ { 2 \pi } \int _ { - L } ^ { L } \left[v_{r}(R, \varphi, z, \tau)-\right.\right.\right. \\
\left.-\bar{v}_{r}(R, \varphi, z, \tau)\right] d z d \varphi(\Theta-t) d t-\frac{1}{\pi \Theta R^{2}} \int_{0}^{\Theta}(\Theta-t) \int_{0}^{R} \int_{0}^{2 \pi} \int_{-L}^{L}\left[v_{r}(r, \varphi, z, t)-\bar{v}_{r}(r, \varphi, z, t)\right] d z d \varphi d r d t \times \\
\left.\left.+\frac{V_{0}}{2}\right\}\left\{\frac{1}{\pi \Theta R L} \int_{0}^{\Theta}(\Theta-t) \int_{0}^{2 \pi} \int_{-L}^{L}\left[v_{r}(R, \varphi, z, \tau)-\bar{v}_{r}(R, \varphi, z, \tau)\right] d z d \varphi d t+1+\frac{\Theta V_{0}}{R L}\right\}^{-1}\right)
\end{gathered}
$$

The second-order approximations of temperature and concentration of gaseous mixture have been calculated framework the method of averaging of function corrections [14-19], i.e. by replacement of the required functions in right sides of equations (12) and (13) on the following sums $T \rightarrow \alpha_{2 T}+T_{1}, C \rightarrow \alpha_{2 C}+C_{1}$. In this case the second-order approximations of the above required functions could be written as

$$
\begin{gathered}
c \cdot T_{2}(r, \varphi, z, t)=\lambda \int_{0}^{t} \frac{\partial^{2} T_{1}(r, \varphi, z, \tau)}{\partial r^{2}} d \tau+\lambda \frac{1}{r^{2}} \int_{0}^{t} \frac{\partial^{2} T_{1}(r, \varphi, z, \tau)}{\partial \varphi^{2}} d \tau+\lambda \int_{0}^{t} \frac{\partial^{2} T_{1}(r, \varphi, z, \tau)}{\partial z^{2}} d \tau+T_{r}+ \\
+\int_{0}^{t} p(r, \varphi, z, \tau) d \tau-c \cdot \frac{\partial}{\partial r} \int_{0}^{t}\left\{\left[v_{r}(r, \varphi, z, \tau)-\bar{v}_{r}(r, \varphi, z, \tau)\right] \cdot\left[\alpha_{2 C}+C_{1}(r, \varphi, z, \tau)\right] \cdot\left[\alpha_{2 T}+T_{1}(r, \varphi, z, \tau)\right]\right\} d \tau- \\
-\frac{c}{r} \frac{\partial}{\partial \varphi} \int_{0}^{t}\left\{\left[\alpha_{2 C}+C_{1}(r, \varphi, z, \tau)\right] \cdot\left[v_{\varphi}(r, \varphi, z, \tau)-\bar{v}_{\varphi}(r, \varphi, z, \tau)\right] \cdot\left[\alpha_{2 T}+T_{1}(r, \varphi, z, \tau)\right]\right\} d \tau- \\
-c \cdot \frac{\partial}{\partial z} \int_{0}^{t}\left\{\left[v_{z}(r, \varphi, z, \tau)-\bar{v}_{z}(r, \varphi, z, \tau)\right] \cdot\left[\alpha_{2 C}+C_{1}(r, \varphi, z, \tau)\right] \cdot\left[\alpha_{2 T}+T_{1}(r, \varphi, z, \tau)\right]\right\} d \tau \\
-c \cdot \frac{\partial}{\partial z} \int_{0}^{t}\left\{\left[v_{z}(r, \varphi, z, \tau)-\bar{v}_{z}(r, \varphi, z, \tau)\right] \cdot\left[\alpha_{2 C}+C_{1}(r, \varphi, z, \tau)\right] \cdot\left[\alpha_{2 T}+T_{1}(r, \varphi, z, \tau)\right]\right\} d \tau \\
C_{2}(r, \varphi, z, t)=\frac{1}{r} \frac{\partial}{\partial r} \int_{0}^{t} r D \frac{\partial C_{1}(r, \varphi, z, \tau)}{\partial r} d \tau+\frac{1}{r^{2}} \frac{\partial}{\partial \varphi} \int_{0}^{t} D \frac{\partial C_{1}(r, \varphi, z, \tau)}{\partial \varphi} d \tau+C_{0} \delta(z+L)+ \\
+\frac{\partial}{\partial z} \int_{0}^{t} D \frac{\partial C_{1}(r, \varphi, z, \tau)}{\partial z} d \tau-\frac{1}{r} \frac{\partial}{\partial r}\left\{r \int_{0}^{t}\left[\alpha_{2 C}+C_{1}(r, \varphi, z, \tau)\right] \cdot\left[v_{r}(r, \varphi, z, \tau)-\bar{v}_{r}(r, \varphi, z, \tau)\right] d \tau\right\}- \\
-\frac{1}{r} \frac{\partial}{\partial \varphi} \int_{0}^{t}\left[\alpha_{2 C}+C_{1}(r, \varphi, z, \tau)\right] \cdot\left[v_{\varphi}(r, \varphi, z, \tau)-\bar{v}_{\varphi}(r, \varphi, z, \tau)\right] d \tau- \\
-\frac{\partial}{\partial z} \int_{0}^{t}\left[\alpha \alpha_{2 C}+C_{1}(r, \varphi, z, \tau)\right] \cdot\left[v_{z}(r, \varphi, z, \tau)-\bar{v}_{z}(r, \varphi, z, \tau)\right] d \tau
\end{gathered}
$$


Averages values of the second-order approximations of temperature and concentration of mixture $\alpha_{2 T}$ and $\alpha_{2 C}$ have been calculated by using the following standard relations

$$
\alpha_{2 T}=\frac{1}{\pi \Theta R^{2} L} \int_{0}^{\Theta} \int_{0}^{R} r \int_{0}^{2 \pi} \int_{-L}^{L}\left(T_{2}-T_{1}\right) d z d \varphi d r d t, \alpha_{2 C}=\frac{1}{\pi \Theta R^{2} L} \int_{0}^{\Theta} \int_{0}^{R} r \int_{0}^{2 \pi} \int_{-L}^{L}\left(C_{2}-C_{1}\right) d z d \varphi d r d t
$$

Substitution of the first- and the second-order approximations of temperature and concentration of mixture into relations (18) gives us possibility to obtain equations to determine required average values

$$
\begin{aligned}
& \alpha_{2 T}=\left(\frac{\lambda \sigma}{c \pi \Theta R L} \int_{0}^{\Theta}(\Theta-t) \int_{0}^{2 \pi} \int_{-L}^{L} T^{4}(R, \varphi, z, t) d z d \varphi d t-\frac{\lambda}{c \pi \Theta R^{2} L} \int_{0}^{\Theta}(\Theta-t) \int_{0}^{2 \pi} \int_{-L}^{L} T_{1}(R, \varphi, z, t) d z d \varphi d t \times\right. \\
& +\frac{\lambda}{c \pi \Theta R^{2} L} \int_{0}^{\Theta}(\Theta-t) \int_{0}^{2 \pi} \int_{-L}^{L} T_{1}(0, \varphi, z, t) d z d \varphi d t-\int_{0}^{\Theta}(\Theta-t) \int_{0}^{2 \pi} \int_{-L}^{L}\left\{T_{1}(R, \varphi, z, t)\left[\alpha_{2 C}+C_{1}(R, \varphi, z, t)\right]-\right. \\
& \left.-\alpha_{1 T} \alpha_{1 C}\right\}\left[v_{r}(R, \varphi, z, t)-\bar{v}_{r}(R, \varphi, z, t)\right] d z d \varphi d t \frac{1}{\pi \Theta R L}-\int_{0}^{\Theta} \int_{0}^{R} r \int_{0}^{2 \pi} \int_{-L}^{L}\left[v_{r}(r, \varphi, z, t)-\bar{v}_{r}(r, \varphi, z, t)\right] \times \\
& \times\left\{T_{1}(r, \varphi, z, t)\left[\alpha_{2 C}+C_{1}(r, \varphi, z, t)\right]-\alpha_{1 T} \alpha_{1 C}\right\} \cdot d z d \varphi d r(\Theta-t) d t \frac{1}{\pi \Theta R^{2} L}-\frac{V_{0}}{\pi \Theta R^{2} L \int_{0}^{\Theta}(\Theta-t) \times} \\
& \int_{0}^{R} r \int_{0}^{2 \pi}\left[T_{1}(r, \varphi, L, t)\left(\alpha_{2 C}+C_{0}\right)-\alpha_{1 T} \alpha_{1 C}\right] d \varphi d r d t\left\{\frac{1}{\pi \Theta R L} \times \int_{0}^{\Theta}(\Theta-t) \int_{0}^{2 \pi} \int_{-L}^{L}\left[\alpha_{2 C}+C_{1}(R, \varphi, z, t)\right] \times\right. \\
& \quad \times\left[v_{r}(R, \varphi, z, t)-\bar{v}_{r}(R, \varphi, z, t)\right] d z d \varphi d t+1-\frac{1}{\pi \Theta R^{2} L \int_{0}^{\Theta}(\Theta-t) \int_{0}^{R} r \int_{0}^{2 \pi} \int_{-L}^{L}\left[\alpha_{2 C}+C_{1}(r, \varphi, z, t)\right] \times} \\
& \left.\times \quad \times\left[v_{r}(r, \varphi, z, t)-\bar{v}_{r}(r, \varphi, z, t)\right] d z d \varphi d r d t+2 \Theta V_{0}\left(\alpha_{2 C}+C_{0}\right) / L\right\}^{-1}, \\
& \alpha_{2 C}=\frac{1}{\pi \Theta R^{2} L} \int_{0}^{\Theta}(\Theta-t) \int_{0}^{R} r \int_{0}^{2 \pi} D\left[\left.\frac{\partial C_{1}(r, \varphi, z, \tau)}{\partial z}\right|_{z=L}-\left.\frac{\partial C_{1}(r, \varphi, z, \tau)}{\partial z}\right|_{z=-L}\right] d \varphi d r d t-\frac{1}{\pi \Theta R^{2} L} \times \\
& \times \int_{0}^{\Theta}(\Theta-t) \int_{0}^{2 \pi} \int_{-L}^{L}\left\{r\left[\alpha_{2 C}-\alpha_{1 C}+C_{1}(R, \varphi, z, \tau)\right] \cdot\left[v_{r}(R, \varphi, z, \tau)-\bar{v}_{r}(R, \varphi, z, \tau)\right]\right\} d z d \varphi d t-\frac{V_{0}}{\pi \Theta R^{2} L} \times \\
& \times \int_{0}^{\Theta}(\Theta-t) \int_{0}^{R} r \int_{0}^{2 \pi}\left(\alpha_{2 C}-\alpha_{1 C}+C_{0}\right) d z d \varphi d r d t .
\end{aligned}
$$

